\title{
NUTZFAHRZEUGTECHNIK FÜR HEUTE UND MORGEN
}

\author{
Die Nutzfahrzeugindustrie steht vor herausfordernden Jahren: Im Spannungsfeld zwischen \\ Wirtschaftswachstum und Klimaschutz müssen Techniken erdacht und realisiert werden, \\ die ökonomische und ökologische Interessen gleichermaßen befriedigen. Die diesjährige \\ Nutzfahrzeug-IAA in Hannover wird so einmal mehr zum Innovationsschaufenster.
}

Seit Beginn der 1990er-Jahre hat die stufenweise Verschärfung der Abgasemissions-Grenzwerte die Entwicklung von schweren Nutzfahrzeugen nachhaltig beeinflusst. Seitdem wurden die Schadstoffemissionen pro Fahrzeug nach Angaben von MAN-Nutzfahrzeugchef Dr. Georg Pachta-Reyhofen um durchschnittlich $85 \%$ gesenkt. Mit der ab dem 1. Januar 2014 obligatorischen Abgasnorm Euro VI werden die Emissionen etwa für $\mathrm{NO}_{\mathrm{x}}$ und Partikel gegenüber 1990 sogar um jeweils bis zu $97 \%$ reduziert.

Allerdings haben die strengen Abgasvorschriften technische Lösungen erzwungen, die einer weiteren Absenkung des Kraftstoffverbrauchs entgegenwirkten. An der Entwicklung der durchschnittlichen Kraftstoffverbräuche schwerer Lkw seit Mitte der 1960erJahre wird dies deutlich: Bis in die 1980er-Jahre hinein sank der Dieselverbrauch von 40-t-Sattelzügen mit jeder neuen Modellgeneration deutlich. Nach Einführung der Euro-Abgasnormen wurden die Fortschritte bei der Kraftstoffverbrauchsminderung trotz erheblicher Einsparbemühungen seitens der Hersteller immer geringer - bis hin zu einer Stagnation.

Diese Situation ist für Klimaschutz, Lkw-Hersteller und die unter einem extremen Kostendruck agierenden Flottenbetreiber gleichermaßen unbefriedigend: Die TCO-Analyse (Total Cost of Ownership) für einen in Deutschland zugelassenen schweren Sattelzug zeigt, dass die Ausgaben für den Dieselkraftstoff mit einem Anteil von knapp $30 \%$ an den gesamten Fahrzeugkosten den größten Kostenblock darstellen - noch vor den Lohn- und Sozialleistungen für das Fahrpersonal. Es ist also nicht nur im Sinne der Klimabilanz entscheidend, dass der Kraftstoffverbrauch von Lkw weiter gesenkt wird, sondern auch im Interesse der Lkw-Betreiber.

Mögliche Stellhebel dafür sind die Elektrifizierung und bedarfsgerechte Steuerung von Nebenaggregat-Antrieben, Thermomanagement (Wärmerekuperation), Hybridisierung, Leichtlaufreifen und permanente Reifendruck-Überwachung, ein intelligentes Verkehrsmanagement und nicht zuletzt eine verbesserte Aerodynamik.

Unser Interviewpartner Bernd Maierhofer, Entwicklungsvorstand bei MAN Trucks \& Bus, findet dazu klare Worte: „Nachdem bei den Emissionen das technisch Mögliche und volkswirtschaftlich Sinnvolle erreicht ist, sollten nun die Anstrengungen auf den Verbrauch refokussiert werden.“

Ein weites Tätigkeitsfeld bietet darüber hinaus auch die Auseinandersetzung mit alternativen Antrieben, die zumindest im Bereich des öffentlichen Personennahverkehrs gar nicht so neu sind, wie ein Blick auf Straßenbahnen und Oberleitungsbusse zeigt. Eine infrastrukturunabhängige Alternative dazu war Ziel des in diesem Heft vorgestellten Smartwheel-Projekts der RWTH Aachen. Dass auch auf Ebene der Komponenten noch Potenziale schlummern, zeigen schließlich die Arbeiten bei Voith Turbo an einem Sekundär-Wasserretarder mit Tempomat-Funktion.
652

Elektrisch angetriebener Minibus für den ÖPNV Felix Töpler, Lutz Eckstein, Gerrit Geulen [RWTH Aachen], Jérôme Homann [fka]

660 „Auf die Verbrauchsreduzierung zurückbesinnen“ Interview mit Bernd Maierhofer [MAN]

664 Neuer Sekundär-Wasserretarder für Busse und Lkw Thomas Ohr, Dieter Laukemann, Tilman Huth [Voith], Frank Steffens [Daimler] 


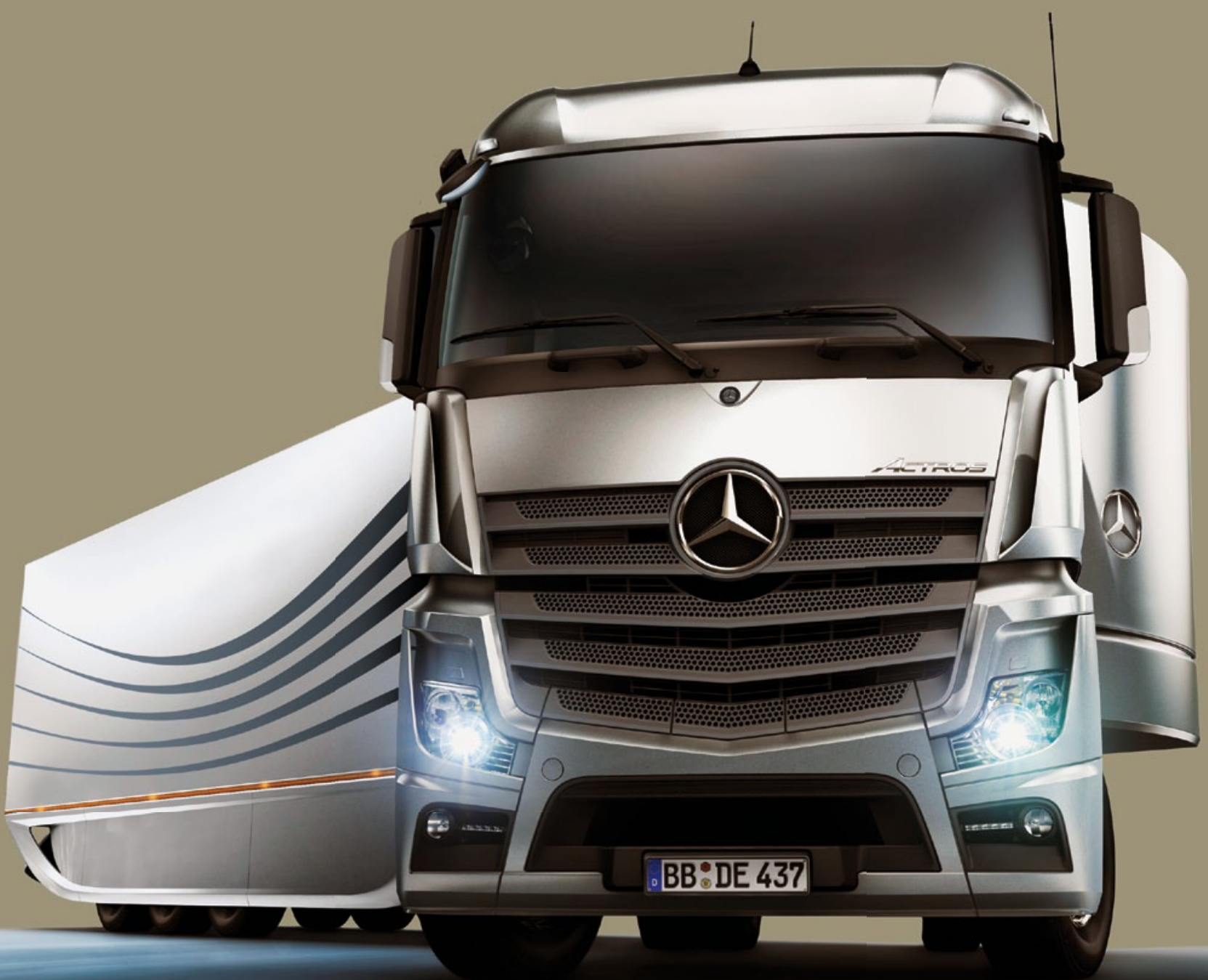

